15

\title{
Влияние геометрии и модового состава излучения на рассеивающие свойства кварц-полимерного оптического волокна со светоотражающей оболочкой Tefzel
}

\author{
(C) A.А. Маковецкий \\ Фрязинский фрилиал Института радиотехники и электроники им. Котельникова РАН, \\ 141190 Фрязино, Московская обл., Россия \\ e-mail: maz226@ms.ire.rssi.ru
}

Поступила в редакцию 01.04.2021 г.

В окончательной редакции 23.04.2021 г.

Принята к публикации 26.04.2021 г.

Для кварц-полимерного оптического волокна с диаметром световедущей сердцевины $400 \mu \mathrm{m}$ и с рассеивающей светоотражающей оболочкой из термопластичного сополимера тетрафторэтилена с этиленом марки Tefzel проведен численный расчет гибридных мод. Для выбранных мод были рассчитаны радиальный профиль и определяющая рассеяние величина $\chi$ - относительная доля энергии моды, распространяющейся по светоотражающей оболочке. Функция $\chi(m, s)$ монотонно возрастает с увеличением азимутального числа $m$ и уменьшается с увеличением радиального числа $s$. Наибольшее значение $\chi$ достигается у моды с максимально возможным для данного оптического волокна значением $m=953$, при котором существует только одна мода с $s=1$. При уменьшении диаметра световедущей сердцевины рассеивание возрастает.

Полученные расчетно-теоретические результаты были качественно подтверждены экспериментами по измерению рассеяния в кварц-полимерных оптических волокнах со светоотражающей оболочкой Tefzel c диаметрами световедущей сердцевины 400 и $200 \mu \mathrm{m}$.

Ключевые слова: многомодовое оптическое волокно, рассеивающая светоотражающая оболочка, характеристическое уравнение, расчет радиальных профилей мод, доля излучения моды, распространяющегося по оболочке.

DOI: $10.21883 / \mathrm{OS} .2021 .09 .51351 .2097-21$

\section{Введение}

Визуально наблюдаемое рассеяние света в оптических волокнах $(\mathrm{OB})$ давно привлекает внимание исследователей с точки зрения создания на его основе гибких протяженных источников света для широкого спектра применений [1-3]. Для создания ОВ с эффективным рассеянием (боковым свечением) используется несколько методов, в том числе многократное микроизгибание оси волокна [4], включение рассеивающих центров в сердцевину [5] или оболочку волокна [3], включение люминесцентных центров в сердцевину волокна [6], радиационное облучение ОВ с германосиликатной сердцевиной [7], создание асимметрии в геометрии сердцевина/оболочка и другие методы.

Наиболее разработанным методом создания преформ рассеивающих кварц-кварцевых ОВ является метод внешнего осаждения - Outside Vapor Deposition (OVD). $\mathrm{C}$ его помощью на поверхность цилиндрического штабика из кварцевого стекла наносят слой стекла с газовыми пузырями. После перетяжки преформы в сердцевине волокна у границы со светоотражающей оболочкой формируются нано- и микроразмерные пузыри, вызывающие рассеяние проходящего по ОВ излучения. Таким методом компания Corning Inc. создала продукт под названием Fibrance TM Light Diffusing Fiber [8]. Рассеивающие пузыри в сердцевине преформы создают также методом модифицированного химического осаждения из паровой фазы (MCVD) [9].

Рассеивающие кварц-полимерные ОВ создают внесением рассеивающих добавок в жидкую полимерную композицию для нанесения светоотражающего покрытия [10].

Заметим, что при исследовании оптических свойств рассеивающих ОВ использовались феноменологические модели, модовый состав излучения не учитывался.

Кварц-полимерное оптическое волокно со светоотражающей оболочкой из сополимера тетрафторэтилена с этиленом марки являются специальным видом кварцполимерных ОВ, у которых светоотражающая оболочка одновременно является и защитной оболочкой. Этот полимер является кристаллизующимся полимером, т.е. имеющими в объеме материала как аморфную фазу (показатель преломления $n=1.393$ ), так и кристаллическую фазу. Последняя состоит из анизотропных кристаллов различной морфологии (кристаллитов, фибрилл, ламелей, сферолитов). Их показатели преломления слегка отличаются от показателя преломления аморфной фазы. Степень кристалличности полимера составляет 40-60\% [11]. Вследствие этого при распространении света по ОВ наблюдается сильное его рассеяние, видимое под любым углом наблюдения (рис. 1). 


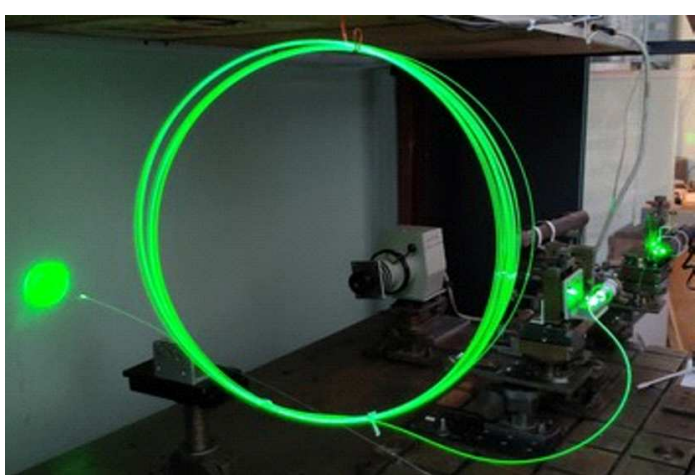

Рис. 1. Фотография бухты ОВ длиной $20 \mathrm{~m}$ (диаметр бухты $220 \mathrm{~mm}$ ) при ее засветке излучением лазера LG Laser 303 c $\lambda=532 \mathrm{~nm}$.

Целью данной работы является теоретическое исследование зависимости эффективности рассеяния излучения от геометрии и модового состава излучения в многомодовых кварц-полимерных ОВ со светоотражающим покрытием Tefzel, а также качественная проверка полученных результатов на экспериментах по измерению рассеяния в данных ОВ.

\section{Характеристическое уравнение и алгоритм его решения}

Собственные числа гибридных мод оптического волокна со ступенчатым профилем показателя преломления с бесконечной оболочкой задаются характеристическим уравнением [12]:

$$
\begin{aligned}
& \left(\frac{1}{w} \frac{K n^{\prime}(m, w)}{K n(m, w)}+\frac{1}{u} \frac{J n^{\prime}(m, u)}{J n(m, u)}\right)\left(\frac{n_{2}^{2}}{w} \frac{K n^{\prime}(m, w)}{K n(m, w)}\right. \\
& \left.\quad+\frac{n_{1}^{2}}{u} \frac{J n^{\prime}(m, u)}{J n(m, u)}\right)-\left(\frac{V^{2}}{u^{2} w^{2}}\right)^{2} \frac{m^{2} \beta^{2}}{k_{0}^{2}}=0
\end{aligned}
$$

где $J n(m, u), K n(m, w), J n^{\prime}(m, u)$ и $K n^{\prime}(m, w)$ - функции Бесселя первого рода, модифицированные функции Бесселя второго рода и их производные по $u$ и $w$ соответственно, $m$ - азимутальное модовое число, $k_{0}=2 \pi / \lambda-$ волновое число в вакууме, $n_{1}$ и $n_{2}-$ показатели преломления сердцевины и оболочки ОВ. Величины $u$ и $w$ связаны с осевой постоянной распространения $\beta$ и приведённой частотой $V$ соотношениями:

$$
\begin{gathered}
u=a\left(n_{1}^{2} k_{0}^{2}-\beta_{2}\right)^{1 / 2}, \quad w=a\left(\beta^{2}-n_{2}^{2} k_{0}^{2}\right)^{1 / 2}, \\
V=a k_{0}\left(n_{1}^{2}-n_{2}^{2}\right)^{1 / 2},
\end{gathered}
$$

где $a-$ радиус сердцевины ОВ.

В качестве переменной выберем величину $u$, выразим через нее величины $w$ и $\beta$. Обозначим левую часть уравнения (1) через $F s(u, m), u \in(0, V)$. Разобьем этот интервал на $N=10^{5}$ равных частей точками с координатами $u_{j}=V(j-1) / N, j=1,2, \ldots, N, N+1$. Построим в системе Mathcad графики функций $\operatorname{sign}\left(F s\left(u_{j}, m\right)\right)$ для ОВ с параметрами: $n_{1}=1.456$ (кварцевое стекло), $n_{2}=1.393$ (Tefzel), $a=200 \mu \mathrm{m}$ (диаметр сердцевины $400 \mu \mathrm{m})$. Графики функций $\operatorname{sign}\left(F s\left(u_{j}, m\right)\right)$, рассчитанные для нескольких значений азимутального числа $m$, приведены на рис. 2. Решения уравнения (1) лежат на каждом из отрезков $\left(u_{j}, u_{j+1}\right)$, в концевых точках которых функция $\operatorname{sign}\left(F s\left(u_{j}, m\right)\right)$ меняет значение с -1 на +1 или наоборот. Определив все такие отрезки для заданного значения азимутального числа $m$, затем с помощью процедуры Mathcad $\operatorname{root}(f(x), x)$ определяли решения $u_{m, s}$, где $s$ - радиальное число.

\section{Качественный анализ решений характеристического уравнения}

Отметим основные свойства решений характеристического уравнения для КП-OB/Tefzel $/ 400 \mu \mathrm{m}$.

1. Интервал решений уравнения (1) при увеличения азимутального числа сужается от $\approx(0, V)$ при $m=1$ до одной точки при $m=m_{\max }=953$.

2. Семейство решений уравнения (1) состоит из пар близко стоящих друг к другу решений, которые на рис. 1 в большинстве своем визуально не различимы.

3. Число гибридных мод уменьшается от 653 мод при $m=1$ до одной моды при $m=953$.

В данной работе не стояла задача исследования надмолекулярной структуры полимерной светоотражающей оболочки. Предполагалось, что она фиксирована, так что интенсивность рассеяния распространяющейся по ОВ моды определялась долей ее энергии $\chi$, распространяющейся по оболочке:

$$
\chi(m, s)=\frac{\int_{a}^{a 1.1}\left|E_{m, s}^{z}(r, \varphi)\right|^{2} r d r}{\int_{0}^{a}\left|E_{m, s}^{z}(r, \varphi)\right|^{2} r d r+\int_{a}^{a 1.1}\left|E_{m, s}^{z}(r, \varphi)\right|^{2} r d r},
$$

где $E_{m, s}^{z}(r, \varphi)$ - поперечный профиль моды с азимутальным числом $m$ и радиальным числом $s$. Выражение для $E_{m, s}^{z}(r, \varphi)$ имеет вид

$E_{m, s}^{z}(r, \varphi)=\exp (i m \varphi)\left\{\begin{array}{l}J n\left(m, u_{m, s} \frac{r}{a}\right), \quad 0 \leq r \leq a, \\ \frac{J n\left(m, u_{m, s}\right)}{K n\left(m, w_{m, s}\right)} K n\left(m, w_{m, s} \frac{r}{a}\right), \quad r \geq a .\end{array}\right.$

Рассчитанные значения величин $\chi\left(u_{m, s}\right)$ для всех мод с азимутальным числом $m=1$ приведены на рис. 3. Видно, что кривая расщепляется на две кривые, соответствующие ЕН- и НЕ-модам ОВ. Верхняя кривая построена для четных значений радиального числа $s$, нижняя - для нечетных. В дальнейшем будем анализировать верхние ветви зависимостей $\chi\left(u_{m, s}\right)$.

На рис. 4 приведены рассчитанные зависимости $\chi\left(u_{m, s}\right)$ для мод КП-ОВ/Tefzel/400 $\mu$ m с другими значениями азимутального числа $m$. Отметим основные свойства приведенных кривых. 

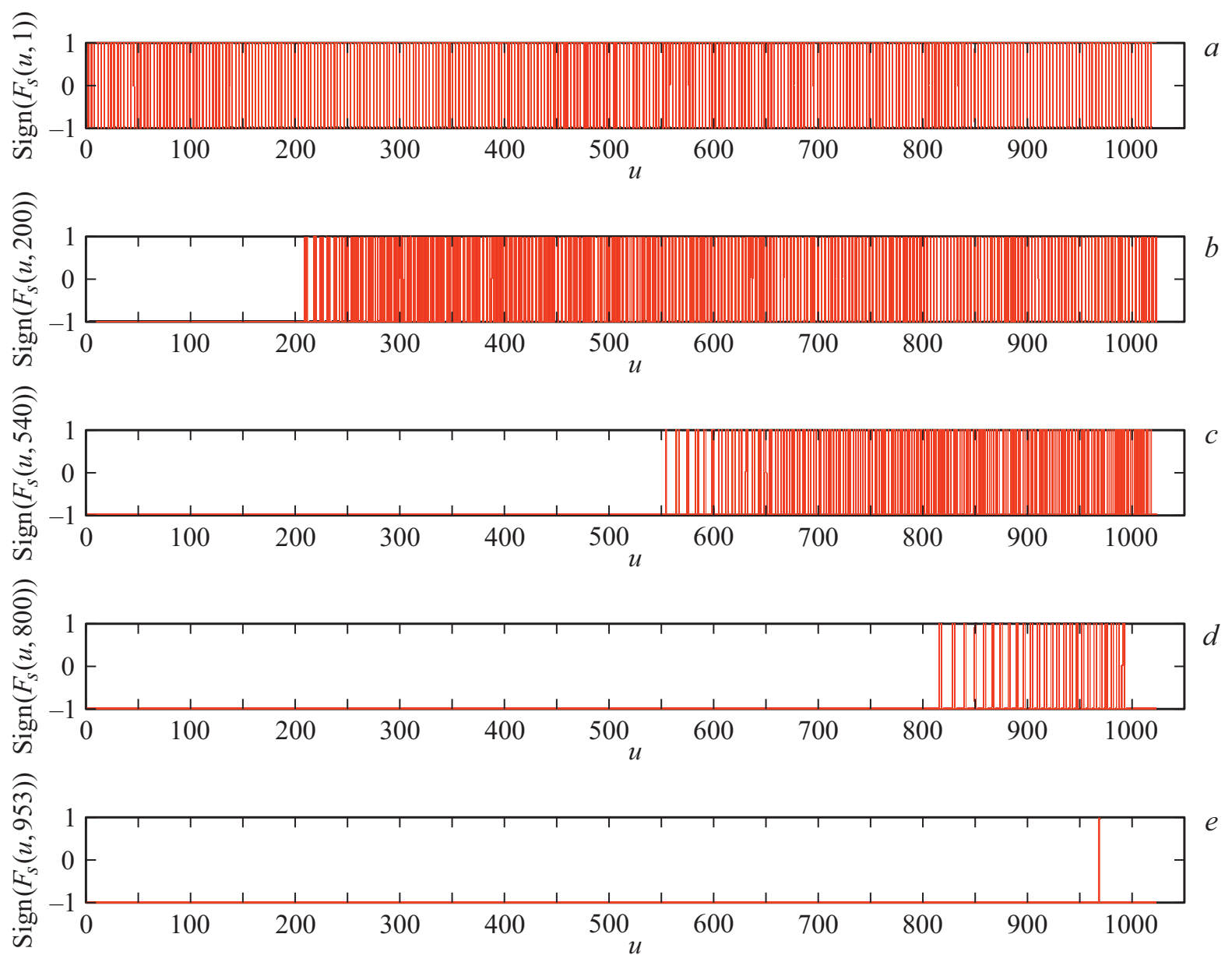

Рис. 2. Графики функций $\operatorname{sign}\left(F s\left(u_{j, m}\right)\right)$, качественно иллюстрирующие количество и расположение гибридных мод КПOB/Tefzel/400 $\mu$ д для $m=1(a), 200(b), 540(c), 800(d)$ и $953(e)$.

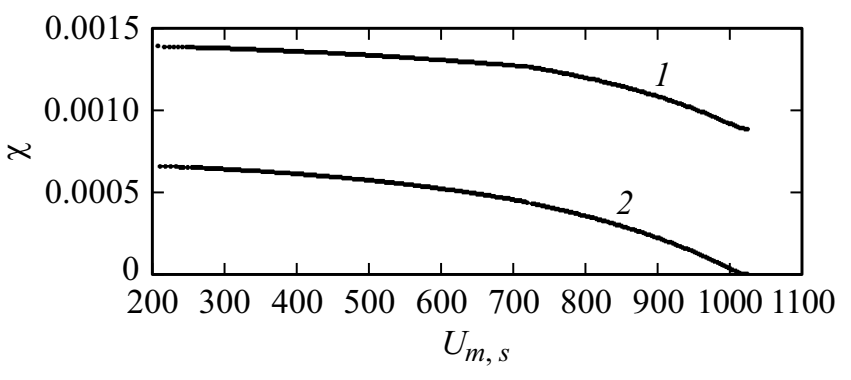

Рис. 3. Рассчитанные значения $\chi\left(u_{m, s}\right)$ гибридных мод КП$\mathrm{OB} / \mathrm{Tefzel} / 400 \mu \mathrm{m}$ при $m=1$ для четных (1) и для нечетных $s$ (2).

1. При любом допустимом значении азимутального числа $m$ наибольшее значение величина $\chi$ достигает при значении радиального числа $s=1$. При увеличении $s$ величина $\chi$ монотонно убывает.

2. При увеличении азимутального числа $m$ величины $\chi$ возрастают, достигая своего максимального значения $\chi_{\max }=3.395 \cdot 10^{-3}$ для $m_{\max }=953$. Отметим, что при $m=m_{\max }$ существует только одна мода.
На рис. 5 изображены рассчитанные радиальные профили нескольких мод КП-ОВ/Tefzel/400 $\mu \mathrm{m}$, поясняющие и дополняющие кривые, представленные на рис. 2. Из них видно, что для мод с радиальным числом $s=1$ радиальные профили представляет собой „одногорбные“ кривые. При $s>1$ профили моды - осциллирующие кривые, с уменьшением амплитуды осцилляций при

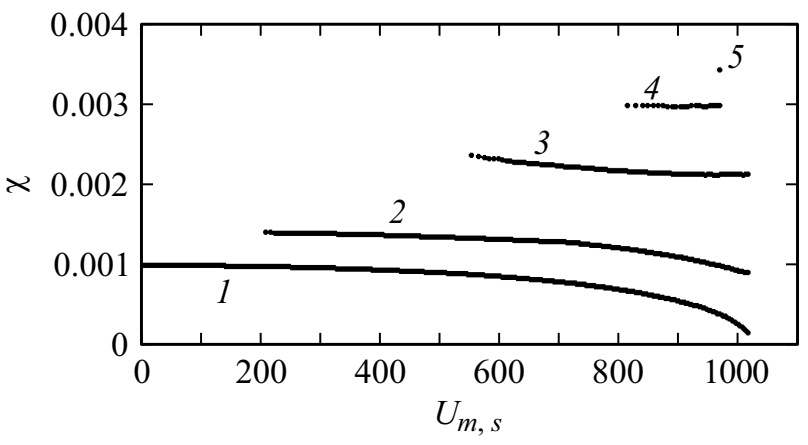

Рис. 4. Рассчитанные значения величин $\chi$ гибридных мод КП$\mathrm{OB} / \mathrm{Tefzel} / 400 \mu \mathrm{m}$ для $m=1$ (1), 200 (2), 540 (3), 800 (4) и 953 (5). 

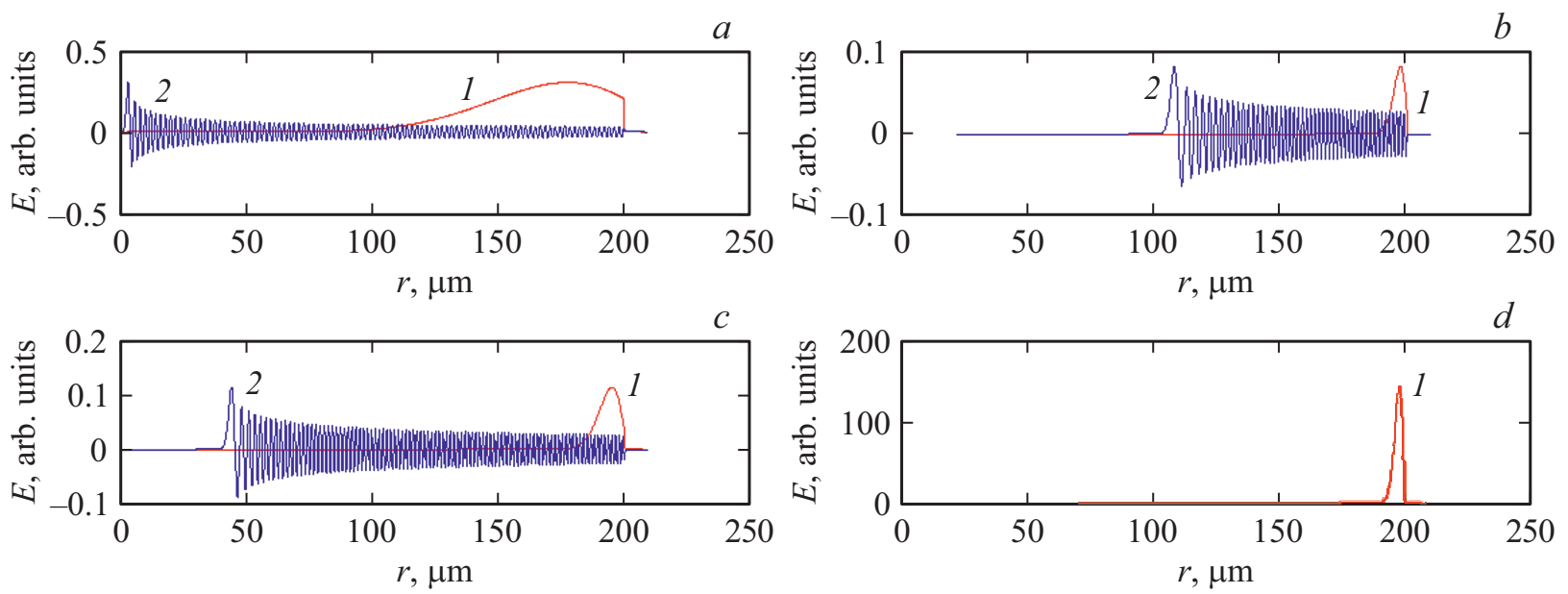

Рис. 5. Рассчитанные радиальные профили гибридных мод КП-ОВ/Tefzel/400 $\mu \mathrm{m}$ для $m=1(a), 200(b), 540(c)$ и 953 (d). Профили с индексами 1 соответствуют $s=1$, с индексами $2-s=s_{\max }$.

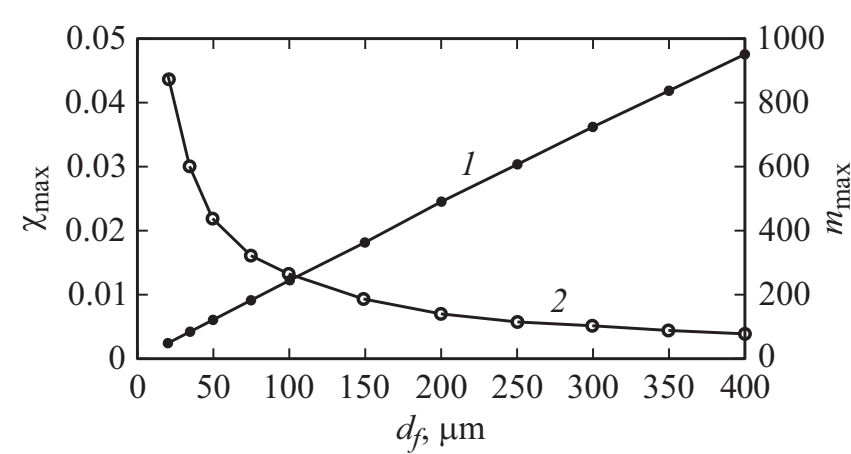

Рис. 6. Рассчитанные зависимости величин $m_{\max }(1)$ и $\chi_{\max }(2)$ от диаметра световедущей сердцевины $d_{f}$ для КП-ОВ/Tefzel/ $d_{f}$.

увеличении расстояния от оси волокна $r$. При $m>1$ в центре волокна образуется темное пятно (характерный признак гибридных мод, оптических вихрей). Это пятно соответствует каустике косых лучей в геометрической оптике. При этом чем больше азимутальное число, тем шире каустика, поле в сердцевине сосредоточивается во все более тонком цилиндре у границы раздела с оболочкой. Наконец, при $m=953$ остается только одна мода с самой широкой каустикой и самым узким цилиндром, в котором она сосредоточена. Именно эта мода имеет наибольшее значение $\chi$ среди всех остальных мод данного ОВ.

Представляет практический интерес изучение зависимости величин $\chi_{\max }$ и $m_{\max }$ от диаметра световедущей сердцевины $d_{f}$. Рассчитанные зависимости приведены на рис. 6. Из них следует, что интенсивность рассеяния излучения в КП-OB/Tefzel $/ d_{f}$ может существенно возрасти при уменьшении диаметра светоотражающей сердцевины $d_{f}$ от 400 до $50 \mu \mathrm{m}$. При этом максимальное значение $\mathrm{m}$ при уменьшении диаметра сердцевины уменьшается.

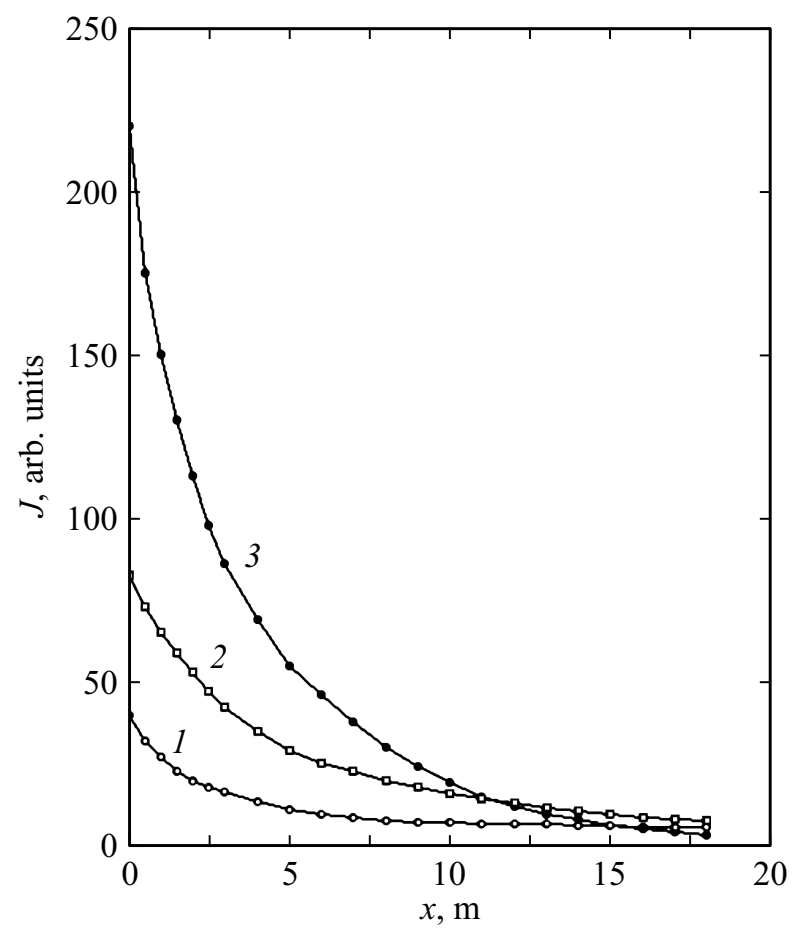

Рис. 7. Распределения интенсивностей бокового рассеяния света $J_{s}(x)$ вдоль оси КП-ОВ/Tefzel $/ 400 \mu \mathrm{m}$ при $\delta=1.5 \mathrm{~mm}$ (1), $3 \mathrm{~mm} \mathrm{(2),} 5 \mathrm{~mm} \mathrm{(3).} \mathrm{Длина} \mathrm{ОВ} 18.5 \mathrm{~m}$, $\lambda=532 \mathrm{~nm}$; шаг измерений $-1 \mathrm{~m}$.

\section{Анализ рассеяния в оптическом волокне КП-ОВ/Tefzel/400 $\mu \mathrm{m}$}

Для возбуждения гибридных мод ОВ сфокусированное излучение лазера LG-303 ( $\lambda=532 \mathrm{~nm})$ необходимо вводить в торец волокна под углом к оси ОВ и со смещением относительно его центра $[13,14]$. Это достигалось поперечным сдвигом $\delta$ оси лазера относительно оси фоку- 


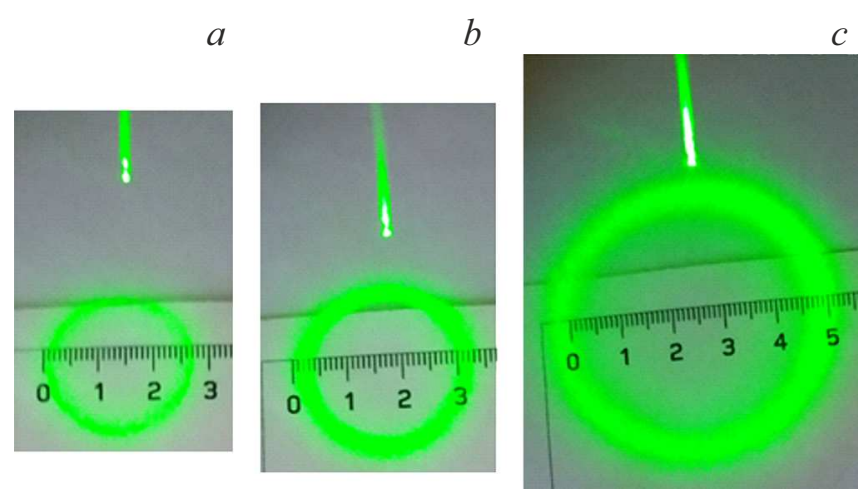

Рис. 8. Фото пятен излучения $\lambda=532 \mathrm{~nm}$ в дальнем поле КПOB/Tefzel $/ 400 \mu \mathrm{m}$ : при $\delta=2.0 \mathrm{~mm}(a), 3 \mathrm{~mm}(b)$ и $5 \mathrm{~mm}(c)$. Расстояние от торца ОВ до экрана $h=48 \mathrm{~mm}$.

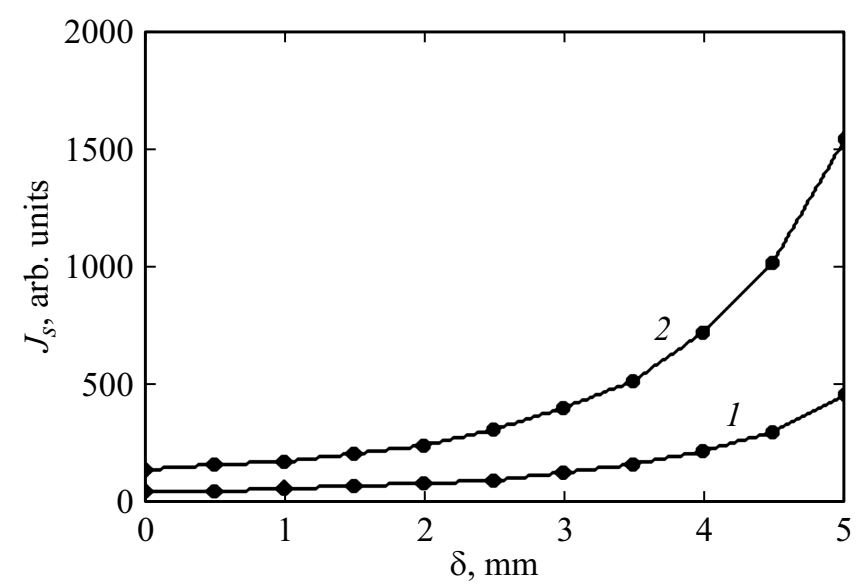

Рис. 9. Зависимости интенсивности бокового рассеяния света $J_{s}$ от величины $\delta$ для КП-ОВ со светоотражающей оболочкой Tefzel для $d_{f}=400 \mu \mathrm{m}(1), 200 \mu \mathrm{m}$ (2). Измерения проведены в начале ОВ $(x=0.5 \mathrm{~m}) ; \lambda=532 \mathrm{~nm}$.

сирующего объектива $10 \times / 0.4$ с помощью механического юстировочного столика. Различные группы гибридных мод возбуждались варьированием величины $\delta$.

Для измерения распределения интенсивности рассеянного излучения вдоль оси ОВ $J_{s}(x)$ с помощью ФЭУ для каждого выбранного расстояния $x$ до входного торца ОВ регистрировалось излучение, рассеянное участком оболочки ОВ длиной $\approx 25 \mathrm{~mm}$. При этом измеряемый участок ОВ устанавливали параллельно светочувствительному торцу ФЭУ на расстоянии $5 \mathrm{~mm}$ от него [15].

Распределения интенсивностей бокового рассеяния света $J_{s}(x)$ вдоль оси ОВ с $d_{f}=400 \mu \mathrm{m}$ для трех значений $\delta$ приведены на рис. 7. Соответствующие им фото пятен излучения, выходящего из ОВ, представлены на рис. 8 . Из рис. 7 следует, что чем больше величина $\delta$ (больше значение азимутального числа $m$ у возбужденных мод), тем интенсивнее было рассеяние и тем быстрее оно ослабевало вдоль оси ОВ. Например, вблизи входного торца ОВ интенсивность рассеяния $J_{s}$ при $\delta=5 \mathrm{~mm}$ была примерно в 6 раз выше, чем при засветке c $\delta=1.5 \mathrm{~mm}$. При этом суммарная (по всей длине $\mathrm{OB}$ ) интенсивность рассеянного оболочкой излучения при $\delta=5 \mathrm{~mm}$ в несколько раз превышала интенсивность излучения, прошедшего через ОВ.

На рис. 9 приведены измеренные зависимости, иллюстрирующие зависимость интенсивности рассеяния КП-OB/Tefzel от диаметра световедущей сердцевины. Из приведенных кривых видно, что у ОВ с диаметром сердцевины $200 \mu \mathrm{m}$ интенсивность рассеяния заметно выше, чем у ОВ с сердцевиной диаметром $400 \mu \mathrm{m}$. Это качественно подтверждает выводы, сделанные в расчетной части статьи.

\section{Заключение}

Сформулируем основные результаты настоящей работы.

1. Численным решением характеристического уравнения проведен расчет гибридных мод (параметров $u_{m, s}$ ) кварц-полимерного оптического волокна со светоотражающей оболочкой Tefzel и с диаметром световедущей сердцевины $400 \mu \mathrm{m}$. Для каждой гибридной моды определена величина $\chi$ - доля энергии, распространяющаяся по оболочке и определяющая интенсивность ее рассеяния.

2. Величина $\chi\left(u_{m, s}\right)$ монотонно возрастает с увеличением азимутального числа $m$ и уменьшается с увеличением радиального числа $s$. Наибольшее значение $\chi$ достигается для моды с максимально возможным для данного оптического волокна значением $m=953$, при котором существует только одна мода со значением $s=1$.

3. Полученные расчетно-теоретические результаты были качественно подтверждены экспериментами по измерению рассеяния в кварц-полимерных оптических волокнах со светоотражающей оболочкой Tefzel c диаметрами световедущей сердцевины 400 и $200 \mu \mathrm{m}$.

\section{Финансирование работы}

Работа выполнена в рамках государственного задания ФИРЭ им. В.А. Котельникова РАН.

\section{Конфликт интересов}

Авторы заявляют, что у них нет конфликта интересов.

\section{Список литературы}

[1] Rawson E.G. // Appl. Opt. 1974. V. 13. N 10. P. 2370.

[2] Arie A., Karoubi R., Gur Y.S., Tur M. // Appl. Opt. 1986. V. 25. N 11. P. 1754.

[3] Spigulis J., Pfafrods D., Stafekis M., Jelinska-Platace W. // Proc. SPIE. 1997. V. 2967. P. 231.

[4] Costello A., Nyikal J., Yu V.Y., McCloud P. // J. Paediatrics and Child Health. 1995. V. 31. N 1. P. 1. 
[5] Logunov S.L., Bennett K.W., Fewkes E.J., Klubben W.S., Paniccia M. // J. Lightwave Technology. 2019. V. 37. N 22. P. 5667-5673. doi 10.1109/JLT.2019.2928697

[6] Кизеветтер Д.В., Славина А.Ю., Левин В.М., Баскаков Г.Г. // Научно-технические ведомости СПбГПУ. 2012. № $1 / 6$. C. 119.

[7] Ероньян М.А., Комаров А.В., Ломасов В.Н., Реуцкий А.А., Унтилов А.А., Устинов С.В. // Сб. трудов Междунар. Конф. Прикладная оптика - 2018. СПб., 19-21 декабря 2018 г. T. 1. C. 134.

[8] Corning Fibrance Light-Diffusing Fibers // http://www.corning.com/corning fibrance light-diffusing fibers/product information sheet

[9] Bisyarin M.A., Eronyan M.A., Kulesh A.Yu., Meshkovskiy I.K., Reutsky A.A., Shcheglov A.A., Ustinov S.V. // J. Opt. Soc. Am. B. 2017. V. 34. N 11. P. 2396. doi 10.1364/JOSAB.34.002396

[10] Lanzarini-Lopes M., Garcia-Segura S., Hristovski K., Messerly M., Simon A.J., Westerhoff P. // J. Opt. Soc. Am. Part B. 2019. V. 36. N 6. P. 1623. doi 10.1364/JOSAB.36.001623

[11] Энциклопедия полимеров. Т. 3. М.: Советская энциклопедия. 1977. $793 \mathrm{c.}$

[12] Snitzer E. // J. Opt. Soc. Am. 1961. V. 51. N 5. P. 491.

[13] Bolshtyansky M.A., Savchenko A.Yu., Zel'dovich B.Ya. // Opt. Lett. 1999. V. 24. N 7. P. 433.

[14] Кизеветтер Д.В., Ильин Н.В. // Научно-технические ведомости СПбГПУ. 2013. № 3(177). С. 151.

[15] Маковеикий А.А., Замятин А.А., Ряховский Д.В. // Радиотехн. и электрон. 2021. Т. 66. № 3. С. 279. doi 10.31857/S0033849421030141 